\title{
Hémihyperplasie corporelle
}

\section{Isolated hemihyperplasia}

\section{S. Faiz $\cdot$ L. Pradeaux $\cdot$ B. Vallé $\cdot$ M. Biais}

Reçu le 2 mars 2013; accepté le 1 avril 2013

(C) SFMU et Springer-Verlag France 2013

Une enfant de six mois, $9 \mathrm{~kg}$, est amenée par sa mère aux urgences pédiatriques pour un syndrome fébrile évoluant depuis $48 \mathrm{~h}$. Au cours de l'examen clinique, on observe une inégalité de longueur et de diamètre d'environ $2 \mathrm{~cm}$ entre les membres inférieurs, la jambe gauche apparaissant plus épaisse et plus longue que la jambe droite (Fig. 1). Cette inégalité est également observée pour l'ensemble de l'hémicorps gauche. Cette asymétrie n'avait pas encore été remarquée, ni par la famille ni par le médecin traitant. L'hémihyperplasie (ou hémihypertrophie) corporelle est évoquée. C'est une anomalie congénitale caractérisée par une croissance asymétrique du crâne, de la face, du tronc, des membres ou de l'hémicorps entier. Elle peut être isolée dite idiopathique ou associée à différents syndromes : syndrome de Beckwith-Wiedemann (associant une macrosomie, une macroglossie, une viscéromégalie et un omphalocèle), syndrome de Klippel-Trénaunay, de Silver-Russel ou de Proteus. L'hémihypertrophie peut être associée à des anomalies dermatologiques (hypertrichose) ou des malformations urogénitales et comporte un risque accru de développement de tumeurs embryonnaires [1]. Un protocole de surveillance doit être mis en place avec dosage de l'alpha-foetoprotéine toutes les 6 à 12 semaines jusqu'à l'âge de quatre ans ainsi qu'une échographie abdominale tous les trois mois jusqu'à trois ans puis tous les six mois jusqu'à la puberté. Les deux tumeurs les plus fréquentes sont l'hépatoblastome et la tumeur de Wilms.

S. Faiz $\cdot$ L. Pradeaux

Service des urgences, Centre hospitalier de Périgueux,

80, avenue Georges Pompidou, F-24000 Périgueux, France

S. Faiz $\cdot$ B. Vallé $(\bowtie) \cdot$ M. Biais

Pôle des urgences adultes, Samu/Smur, CHU de Bordeaux, hôpital Pellegrin, Place Amélie Raba-Léon,

F-33000 Bordeaux, France

e-mail : baptiste.valle@chu-bordeaux.fr

M. Biais

Université Bordeaux Segalen, rue Léo Saignat,

F-33000 Bordeaux, France

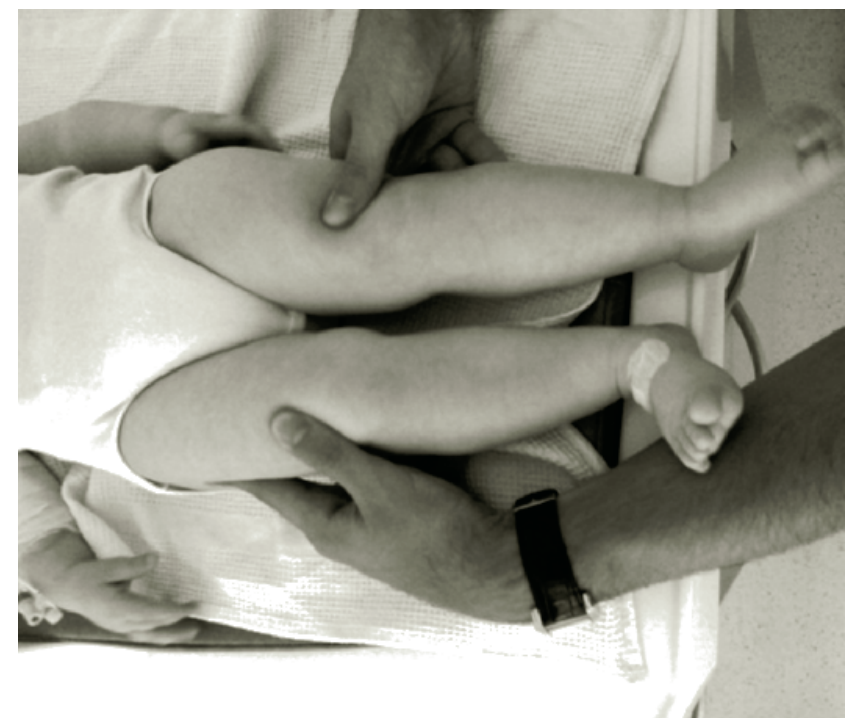

Fig. 1 Le membre inferieur gauche est plus long et plus épais que le membre inferieur droit

Conflit d'intérêt : les auteurs ne déclarent pas de conflit d'intérêt.

\section{Référence}

1. Shuman C, Smith AC, Steele L, et al (2006) Constitutional UPD for chromosome $11 \mathrm{p} 15$ in individuals with isolated hemihyperplasia is associated with high tumor risk and occurs following assisted reproductive technologies. Am J Med Genet 140:1497-503 